\title{
Evaluación Neuropsicológica en educación
}

\author{
Ángela Osuna. Universidad Francisco de Vitoria \\ Recepción: 10 de Junio de 2017 | Aceptado: 16 de Junio de 2017 \\ Correspondencia: Ángela Osuna | Correo-e: a.osuna.prof@ufv.es \\ (iD) 0000-0002-2806-7704 \\ Citar: Osuna, A. (2017). Evaluación Neuropsicológica en educación. ReiDoCrea, 6(2), 24-30.
}

Resumen: En las últimas décadas ha existido un interés creciente por el campo de la neuropsicología, y más concretamente, por el de las evaluaciones neuropsicológicas. Uno de los ámbitos en los que las evaluaciones neuropsicológicas han tenido más auge en los últimos años es el ámbito educativo. El objetivo de estas evaluaciones es conocer la capacidad intelectual de los niños y adolescentes, las capacidades superiores que éstos utilizan durante su aprendizaje (atención y memoria, entre otras) y las posibles alteraciones neuropsicológicas que pueden tener. Este conocimiento favorece el diagnóstico precoz y la implantación de programas psicoeducativos que promuevan el desarrollo académico y neuropsicológico de niños y adolescentes. Uno de los instrumentos neuropsicológicos que mejor evalúa las capacidades cognitivas que utilizan los niños y adolescentes durante su aprendizaje es La Escala de inteligencia de Wechsler para niños (WISC). En este trabajo se hablará de la cuarta edición de esta Escala (WISC-IV), puesto que ha sido la más utilizada en los últimos años. Se comentarán las pruebas e índices de la Escala, las capacidades que evalúa y su gran importancia en la identificación y detección de niños y adolescentes con altas capacidades o con alguna lesión neuropsicológica.

Palabras clave: Evaluación neuropsicológica | Escala de inteligencia de Wechsler para niños

\section{Neuropsychological evaluation in education}

Abstract: During the last few decades there has been an increasing interest in the field of neuropsychology, and more specifically, in neuropsychological assessments. One of the areas in which the neuropsychological assessment have had more interest in recent years is the educational field. The objective of these assessments is to know the intellectual skill of children and adolescents, the superior capacities they use during their learning (attention and memory, among others) and possible neuropsychological alterations that they may have. This knowledge favors the early diagnosis and the implementation of psychoeducational programs that promote the academic and neuropsychological development of children and adolescents. One of the neuropsychological tools that best assesses the cognitive abilities that children and adolescents use during their learning is the Wechsler Intelligence Scale for Children (WISC). In this paper we will talk about the fourth edition of this Scale (WISC-IV), since it has been the most used in recent years. The tests and indexes of the Scale will be discussed, the capacities that evaluate and their great importance in the identification and detection of children and adolescents with high capacities or with some neuropsychological injury.

Keywords: Neuropsychological assessment | Wechsler Intelligence for children

\section{Introducción}

En los últimos años ha existido un interés cada vez mayor por las aplicaciones de la neuropsicología al ámbito educativo. Conocer el nivel intelectual de los niños y adolescentes, así como las capacidades que éstos utilizan durante su aprendizaje es un tema que ha preocupado a las neurociencias desde hace algunos años. Cada vez se realizan más evaluaciones neuropsicológicas y se promueven programas psicoeducativos para niños y adolescentes que presentan alguna dificultad de aprendizaje. 
A lo largo de los años, la evaluación psicológica de niños y adolescentes en edad escolar se ha centrado, en la mayoría de los casos, en su capacidad intelectual. El término inteligencia ha suscitado un gran interés y numerosos estudios y teorías, pero aún hoy en día sigue siendo objeto de estudio.

La capacidad intelectual de los niños y adolescentes está mediada por una serie de capacidades cognitivas que éstos utilizan durante su aprendizaje, como la atención, la memoria y la función ejecutiva. El correcto desarrollo y funcionamiento de estas capacidades favorece el desarrollo intelectual así como su capacidad de aprendizaje.

\subsection{Atención}

Desde el punto de vista neuropsicológico, Muñoz - Céspedes y Tirapu (2001) proponen que la atención se puede definir como una función cerebral que selecciona aquellos estímulos útiles para realizar una actividad mental de entre el conjunto de estímulos que llegan de manera continuada a nuestro cerebro.

Actualmente, no existe una única definición de atención, sino que cuando hablamos de ellas no nos referimos a un proceso unitario, sino a algo constituido por diferentes procesos y niveles. Sohlberg y Mateer (1989) proponen cinco niveles de atención: atención focalizada, atención sostenida, atención selectiva, atención alternante y atención dividida.

\subsection{Memoria}

La memoria, en concreto la memoria de trabajo, es otra capacidad que juega un papel fundamental en el aprendizaje de los niños y adolescentes.

La memoria de trabajo es un sistema que permite "mantener y manipular temporalmente la información que necesitamos para llevar a cabo tareas de aprendizaje, comprensión y razonamiento" (Elosúa, Menor y Peraita, 2001, p. 45).

El modelo de Baddeley y Hitch (1974) establece que la memoria de trabajo está compuesta por tres componentes: lazo fonológico, agenda visuoespacial y ejecutivo central. El primero de ellos, se encarga de procesar la información verbal (Muñoz Céspedes y Tirapu, 2005). El segundo se encarga de codificar la información visual y/o espacial, almacenar esta información de manera transitoria y manipular la información almacenada (Elosúa, Menor y Peraita, 2005). Por su parte, el ejecutivo central coordina la actividad de los otros dos módulos y se encarga de supervisar la actividad cognitiva, coordinar la información, manipularla y planificar actividades futuras (Elosúa, Menor y Peraita, 2005).

Posteriormente, Baddeley (2000) introdujo otro componente más a su modelo de memoria operativa (Modelo multicomponente de la memoria de trabajo): el búfer episódico, un sistema de almacenamiento que trabaja al mismo tiempo con la información proveniente del bucle fonológico, la que proviene de la agenda visuoespacial y la que se encuentra almacenada en la memoria a largo plazo.

\subsection{Función ejecutiva}

Barceló y Periáñez (2004), definen las funciones ejecutivas como un grupo de procesos relacionados con actividades como la resolución de problemas, la planificación, la iniciación de conductas, la estimación cognitiva, la inhibición o la 
memoria prospectiva. Son "el resultado de un sistema supramodal de procesamiento múltiple" (Muñoz Céspedes y Tirapu, 2005, p. 476), es decir existe una relación recíproca entre las funciones ejecutivas, la atención y la memoria.

Respecto a la evaluación propiamente dicha, desde hace varias décadas, los instrumentos de evaluación que se han utilizado para medir la inteligencia de los niños, sobre todo en el ámbito escolar, se basaban en tareas de tipo numérico o espacial. Una de las razones de la medición de la inteligencia a través de tareas numéricas es que algunos autores argumentan que a partir de los cuatro o seis años, los niños son capaces de representar números (Cantlon y Brannon, 2007). Es más, otros autores consideran que los bebés utilizan distintos sistemas para manejar números (Flombaum, Junge y Hause, 2005) gracias a una representación numérica prelingüística heredada filogenéticamente (Brannon, 2006).

\section{Escala de Inteligencia de Wechsler para niños - IV}

Actualmente, uno de los instrumentos más utilizados para medir la capacidad intelectual de niños y adolescentes en edad escolar, así como las capacidades cognitivas que influyen en su aprendizaje es la Escala de Inteligencia de Wechsler para niños.

\section{1. Pruebas e índices}

La Escala de Inteligencia de Wechsler para niños - IV (WISC - IV) es una adaptación de las anteriores escalas de Wechsler (Wechsler, 2003).

EI WISC-IV, de aplicación individual, evalúa la capacidad intelectual y capacidades cognitivas en niños y adolescentes de entre 6 años y 0 meses hasta 16 años y 11 meses (Wechsler, 2003).

EI WISC-IV está compuesto por quince tests de los cuales, cinco son de aplicación opcional. Dichos tests, se mencionan a continuación en el orden que se aplican: Cubos, Semejanzas, Dígitos, Conceptos, Claves, Vocabulario, Letras y números, Matrices, Comprensión, Búsqueda de símbolos, Figuras incompletas, Animales, Información, Aritmética y Adivinanzas. Los últimos 5 tests (Figuras incompletas, Animales, Información, Aritmética y Adivinanzas) son de aplicación opcional (Wechsler, 2003).

Los tests mencionados en el párrafo anterior están agrupados en cuatro índices: Comprensión verbal, Razonamiento perceptivo, Memoria de trabajo y Velocidad de procesamiento. Además, el WISC - IV ofrece un quinto índice: capacidad intelectual total (CIT) (Wechsler, 2003).

En la tabla 1 se presentan los quince test agrupados por índices.

Tabla1.

Tests e índices del WISC - IV

\begin{tabular}{ll}
\hline & Semejanzas \\
Comprensión verbal & Vocabulario \\
& Comprensión \\
& Información \\
& Adivinanzas \\
\hline
\end{tabular}




\begin{tabular}{ll}
\hline & Cubos \\
Razonamiento perceptivo & Conceptos \\
& Matrices \\
& Figuras incompletas \\
\hline \multirow{2}{*}{ Memoria de trabajo } & Dígitos \\
& Letras y números \\
\hline \multirow{2}{*}{ Velocidad de procesamiento } & Aritmética \\
& Claves \\
& Búsqueda de símbolos \\
\hline
\end{tabular}

Fuente: Adaptado de Wechsler (2003)

En la tabla 2, se pueden observar las capacidades cognitivas que evalúan cada uno de los cuatro índices, con sus abreviaturas correspondientes.

Tabla 2.

Capacidades cognitivas evaluadas por cada índice del WISC - VI

\begin{tabular}{ll}
\hline & Aptitudes verbales \\
Comprensión verbal (CV) & Formación de conceptos verbales \\
& Establecimiento de relaciones entre \\
& Conceptos \\
& Comprensión auditiva \\
& Comprensión social \\
& Conocimientos adquiridos \\
\hline & Razonamiento perceptivo \\
& Organización perceptiva \\
& Habilidad visoconstructiva \\
Razonamiento perceptivo (RP) & Análisis visual \\
& Formación de conceptos no verbales \\
\hline & Capacidad para retener en la memoria \\
& determinada información con el objetivo \\
& de manipularla (manipulación mental), \\
para llegar a una solución concreta \\
Atención \\
Concentración \\
Memoria de trabajo \\
\hline Capacidad para focalizar la atención e \\
informar del tiempo que se tarda en \\
completar una actividad concreta. \\
Velocidad de procesamiento mental \\
Velocidad de procesamiento grafomotriz \\
\hline
\end{tabular}

Fuente: Adaptado de Wechsler (2003)

En la tabla 3, se pueden observar las capacidades cognitivas que evalúan cada uno de los quince tests, con sus abreviaturas correspondientes.

Tabla 3. 
Capacidades cognitivas evaluadas por cada test del WISC - VI

\begin{tabular}{|c|c|}
\hline Semejanzas (S) & $\begin{array}{l}\text { Capacidad de abstracción } \\
\text { Formación de conceptos verbales } \\
\text { Memoria semántica } \\
\text { Expresión verbal }\end{array}$ \\
\hline Vocabulario (V) & $\begin{array}{l}\text { Memoria semántica } \\
\text { Fluidez verbal }\end{array}$ \\
\hline Comprensión (C) & $\begin{array}{l}\text { Razonamiento } \\
\text { Fluidez verbal } \\
\text { Capacidad para resolver situaciones } \\
\text { sociales (juicio social) }\end{array}$ \\
\hline Información (I) & $\begin{array}{l}\text { Adquisición y recuperación de } \\
\text { conocimientos relacionados con hechos } \\
\text { generales (memoria semántica) } \\
\text { Fluidez verbal }\end{array}$ \\
\hline Adivinanzas (Ad) & $\begin{array}{l}\text { Razonamiento } \\
\text { Abstracción verbal } \\
\text { Habilidad para relacionar diferentes tipos } \\
\text { de información y crear conceptos } \\
\text { alternativos }\end{array}$ \\
\hline Cubos (CC) & $\begin{array}{l}\text { Percepción visual } \\
\text { Establecimiento de relaciones espaciales } \\
\text { Coordinación visomotriz }\end{array}$ \\
\hline Conceptos (Co) & $\begin{array}{l}\text { Razonamiento no verbal } \\
\text { Formación de categorías }\end{array}$ \\
\hline Matrices (M) & $\begin{array}{l}\text { Fundamentalmente } \\
\text { abstracto }\end{array}$ \\
\hline Figuras incompletas $(\mathrm{FI})$ & $\begin{array}{l}\text { Organización perceptiva } \\
\text { Concentración }\end{array}$ \\
\hline \multirow{2}{*}{ Dígitos (D) } & $\begin{array}{l}\text { En orden directo: amplitud atencional y } \\
\text { codificación. }\end{array}$ \\
\hline & $\begin{array}{l}\text { En orden inverso: control atencional y } \\
\text { memoria de trabajo }\end{array}$ \\
\hline Letras y números (LN) & Fundamentalmente memoria de trabajo \\
\hline Aritmética $(A)$ & $\begin{array}{l}\text { Atención } \\
\text { Memoria a corto plazo } \\
\text { Memoria de trabajo } \\
\text { Representación mental }\end{array}$ \\
\hline Claves $(\mathrm{Cl})$ & $\begin{array}{l}\text { Velocidad de procesamiento } \\
\text { Memoria a corto plazo } \\
\text { Atención. } \\
\text { Coordinación motriz }\end{array}$ \\
\hline Búsqueda de símbolos (BS) & $\begin{array}{l}\text { Velocidad de procesamiento } \\
\text { Atención selectiva } \\
\text { Coordinación visomotora }\end{array}$ \\
\hline Animales (An) & $\begin{array}{l}\text { Velocidad de procesamiento } \\
\text { Habilidades de rastreo visual como } \\
\text { estrategia de búsqueda }\end{array}$ \\
\hline
\end{tabular}

Fuente: Adaptado de Wechsler (2003) 


\section{2. EI WISC-IV en poblaciones con circunstancias particulares.}

En este punto se van a comentar algunos hallazgos relacionados con la aplicación del WISC - IV en poblaciones con circunstancias particulares: niños con altas capacidades, niños con retraso mental leve o moderado, niños con trastornos de aprendizaje, niños con trastorno por déficit de atención e hiperactividad, niños con trastorno del espectro autista y niños con síndrome de Asperger (Wechsler, 2003).

Algunos estudios han demostrado que los niños con altas capacidades obtienen puntuaciones significativamente superiores a niños sin altas capacidades, tanto test de tipo verbal, como en tests de tipo no verbal y en la puntuación de capacidad intelectual total. Algunas investigaciones sugieren que aunque el rendimiento es significativamente superior en todos los casos, en algunos de ellos existen discrepancias entre las puntuaciones de tests de tipo verbal y las puntuaciones de tests de tipo no verbal (Wechsler, 2003).

En el caso de niños con retraso mental, se ha observado que los niños con retraso mental leve obtienen puntuaciones significativamente menores en todos los índices que los niños sin retraso mental. Los niños con retraso mental moderado obtienen puntuaciones menores en todos los índices en comparación con los niños con retraso mental leve. Respecto a los tests, los niños con retraso mental leve y moderado obtienen puntuaciones inferiores a las obtenidas por el grupo control (Wechsler, 2003).

En el caso de niños con trastorno del aprendizaje, los estudios han demostrado que los niños con trastorno de la lectura obtienen puntuaciones menores en todos los índices en comparación con niños sin trastorno de lectura. Los estudios también han demostrado que en el caso de niños trastorno de la lectura y de la expresión escrita, las diferencias de medias de todos los índices, exceptuando el índice RP, fueron significativas. En el caso de niños con trastorno de cálculo, las puntuaciones medias de todos los índices también son significativamente menores a excepción del índice VP. Por último, en el caso de los niños con trastorno de la lectura, de la expresión escrita y el cálculo, las puntuaciones en todos los índices son inferiores en comparación con las obtenidas por el grupo control (Wechsler, 2003).

En el caso de los niños con trastorno por déficit de atención e hiperactividad se ha observado un tamaño del efecto moderado en el índice VP y un tamaño del efecto menor en los índices CV, MT y CIT (Wechsler, 2003).

Algunos estudios han demostrado que los niños con trastorno del espectro autista obtienen puntuaciones significativamente menores en todos los índices en comparación con el grupo control, y un funcionamiento intelectual general menor. Respecto a los tests, las diferencias de medias entre todas las puntuaciones escalares, excepto Cubos y Aritmética, son significativas (Wechsler, 2003).

En el caso de los niños con síndrome de Asperger, se observa un gran tamaño del efecto en VP y un tamaño del efecto menor en MT y CIT (Wechsler, 2003).

Los datos anteriores muestran que el WISC - IV es un instrumento importante en la identificación de niños con diferentes alteraciones. 
Barceló, F. y Periáñez, J. A. (2004). Electrofisiología de las funciones ejecutivas. Revista de neurología, 38 (4), 359 - 365.

Brannon, E. (2006). The representation numerical magnitude. Current Opinion in Neurobiology, 16, 222-229.

Cantlon, J. y Brannon, E. (2007). How much does number matter to a monkey (macaca mulatta)? Journal of Experimental Psychology: Animal Behavior Processes, 33 (1), 32-41.

Elosúa, R., Menor, J. y Peraita H. (2001). Trastornos de memoria en la enfermedad de Alzheimer. Madrid: Trotta.

Flombaum, J., Junge, J. y Hauser, M. (2005). Rhesus monkeys spontaneously compute addition operations over large numbers. Cognition, 97 (3), 15-325.

Muñoz Céspedes, J. M. y Tirapu, J. (2001). Rehabilitación neuropsicológica. Madrid: Síntesis.

Muñoz Céspedes, J.M. y Tirapu, J. (2005). Memoria y funciones ejecutivas. Revista de neurología, 41 (8), 475 - 484.

Sohlberg, M. M. y Mateer, C. A. (1989). Introduction to cognitive rehabilitation: Theory \& practice. Nueva York: Guilford Press.

Wechsler, D. (2003). Escala de inteligencia de Wechsler para niños - IV (WISC - IV). Manual técnico y de interpretación. Bloomington: NCS Pearson Inc. 